\title{
Image Analysis Protocol for Detecting and Counting Viable and Inviable Pollen Grains
}

\author{
Samuel J. Mudd ${ }^{1} \&$ H. S. Arathi ${ }^{1}$ \\ ${ }^{1}$ Department of Biology, Colorado State University, USA \\ Correspondence: H. S. Arathi, Department of Biology, Colorado State University, USA. Tel: 1-970-491-0952. \\ E-mail: arathi@lamar.colostate.edu
}

Received: April 16, 2012 Accepted: May 18, 2012 Online Published: August 20, 2012

doi:10.5539/jps.v1n2p158 URL: http://dx.doi.org/10.5539/jps.v1n2p158

\begin{abstract}
Estimating total pollen number and viability is labor intensive and time consuming. Nevertheless the information is crucial for a range of plant biologists from plant breeders to evolutionary biologists. Viability is determined by dye staining and counting colored (viable) and transparent (inviable) grains under a compound microscope. Existing protocols have been standardized to speed up total pollen counts but their success in determining viability is rather limited because they do not incorporate staining techniques. Some of the published protocols that determine viability do so based on statistical methods but focus on just one parameter such as pollen diameter requiring manual standardization to generate two distinct size distributions for viable and inviable pollen. We demonstrate a digital image processing protocol that can count viable and inviable pollen by distinguishing colored and transparent objects in the image space, saving valuable labor and time. Using pollen grains from two plants, Collinsia heterophylla and Brassica napus, we show that differences between viable and inviable pollen are best described by a complex measure, the grain shape. By measuring several parameters such as area, length, width, circularity and elongation, while retaining all the advantages of traditional staining process, our procedure increases the accuracy of viability estimates. The only drawback of our protocol is that it uses the NIS elements a software_specific to Nikon instruments.
\end{abstract}

Keywords: Brassica napus, Collinsia heterophylla, imaging software, pollen count, pollen viability

\section{Introduction}

One of the routine tasks in plant evolutionary biology, pollination biology and crop breeding research is determining male fitness functions by quantifying total pollen grains per flower and proportion of viable pollen (Dafni \& Firmage, 2000; Dafni, Kevan, \& Husband, 2005; Fang, Turner, Yan, Li, \& Siddique, 2010; Kearns \& Inouye, 1993). Typically these data are obtained by manual counts of pollen grains following the process of staining to differentiate between viable and inviable pollen (Herlihy \& Delph, 2009; Heslop-Harrison, Heslop-Harrison, \& Shivanna, 1984; Prasad, Craufurd, \& Summerfield, 1999). The common methods for obtaining pollen counts thus include (i) manual counting under a microscope using a haemocytometer (slides with grids that accommodate a specified volume of the solution), (ii) electronic particle counters and (iii) image processing software. Although manual counting yields highly repeatable and accurate results, it is also extremely tedious, time consuming and labor intensive, requiring each sample to be counted twice (once for viable and again for inviable pollen). Flow cytometry methods have been described to determine pollen viability based on the nuclear DNA content in mature pollen grains but this is a fairly lengthy and expensive process not suitable for a quick estimate (Bino, Tuy, \& De Vries, 1990). While a few recent articles describe alternative digital methods for counting pollen, but without regard to viable and inviable grains (Bechar et al., 1997; Costa \& Yang 2009), there is one study that describes the use of particle counters to estimate pollen viability based on variation in pollen size (Kelly, Rasch, \& Kalisz, 2002) and another that uses fluorescence microscopy by Aronne, Cavuoto and Eduardo (2001).

The use of particle counters for distinguishing viable from inviable pollen grains skips the need for staining of pollen grains because the counters can differentiate objects based on size. This method however, has to be preceded by a preliminary study that measures the size of viable and inviable pollen in each of the target species by staining and manual counting (Kelly et al., 2002). This preliminary step generates two distinct size distributions for viable and inviable pollen and determines the extent of overlap in their diameters. However, 
inviable pollen grains even within the same species generally come in different shapes owing to changes in circularity and elongation during micro-gametogenesis (Barrett, 2002) but particle counters cannot take these differences into consideration. The counters require a pre-set aperture for a given shape and a narrow size rangeand can therefore only distinguish pollen based on the diameter. Thus, particle counters are likely to miss many pollen grains, especially the inviable ones that do not fit the defined size measures.

With increasing pervasiveness of digital imaging, software capacities to process these images continue to gain popularity (Aronne et al., 2001; Fonseca, Westgate, \& Doyle, 2002; France, Duller, Duller, \& Lamb, 2000). The protocol described in a recent article by Costa and Yang (2009) provides evidence for increased accuracy while simultaneously reducing the time taken to determine total pollen content per flower even though it did not utilize the ability of light microscopes to distinguish objects based on their color spectrum. The purpose of our study was to combine the benefits of using stains to differentiate the quality of pollen grains and applying image analysis protocol to count stained and unstained pollen grains. Our procedure takes advantage of the software's intrinsic ability to distinguish particles based on their color, shape and size without the need for fluorescence microscopy as in Aronne et al. (2001). We test the validity of our protocol using pollen grains of two plant species from distinctly different families, Collinsia heterophylla (Fam. Plantaginaceae) and Brassica napus (Fam. Brassicaceae). Both these species produce copious pollen grains with variable proportions of inviable pollen, making their manual counting a tedious task.

\section{Materials and Methods}

\subsection{Preparation of Pollen Samples}

Flowers for pollen counts were collected at peak anther maturity (i.e. when all anthers have dehisced). Anthers were collected using a pair of sharp forceps and carefully deposited into a centrifuge tube that was kept open for a week allowing anthers to air dry. 50 $\mu \mathrm{L}$ of aniline blue stain in lactophenol (PML Microbiologicals, Wilsonville, OR. USA) was added into each of the centrifuge tubes containing the dried anthers. The centrifuge tube was vortexed at medium speed for 30-45 seconds allowing for uniform dispersal of the pollen grains in the stain solution. Two samples, $10 \mu \mathrm{L}$ each, of the pollen-stain solution were placed on separate microscope slides and a $22 \times 22 \mathrm{mM}$ cover slip was dropped on the sample taking care to minimize air bubbles under the cover slip.

\subsection{Imaging Methods}

Nikon Eclipse 80i compound microscope (Nikon instruments Inc. Melville, NY), and NIS-Elements Imaging Software were used for imaging and counting the pollen grains. For best pollen visibility, the light was set at $40 \%$ of the maximum brightness, making the image background tan colored (Figure 1a) and providing a distinct contrast to the pollen grains. The microscope focus was adjusted until all the stained viable pollen grains were clearly visible at $10 \mathrm{X}$ magnification. In order to obtain pollen counts, an image of a random area representing $0.5 \%$ of the total area under the cover slip was captured and digitally processed for each replicate. Two such images were considered sufficiently representative based on the similarity of extrapolated numbers from the images to that obtained from total counts of pollen grains following organized counting from left to right throughout the entire area under the cover slip.

\subsection{Image Processing for Viable (Stained) Pollen Count}

The NIS-Elements Imaging Software can count objects in an image based on their color and shape (Figure 1) once the image is processed in the following manner (for detailed steps see Appendix 1). First, the image background was altered to a dull yellow color allowing the viable blue pollen grains to appear black (Figure 1b). The image contrast was then adjusted such that the viable pollen grains appear solid black in a bright yellow background (Figure 1c). Using the 'clean', 'smooth' and 'separate' functions of the software program, the viable pollen was made to appear distinct from other extraneous material. Placing the crosshair reticle of the image analysis program on a viable pollen grain, the length, width, area, circularity and elongation settings were now adjusted so that all the stained pollen grains with similar dimensions are picked up by the software. Once a set of measurements could isolate all the viable pollen grains, it was saved as 'viable pollen count settings' for use with other slides during software training. 

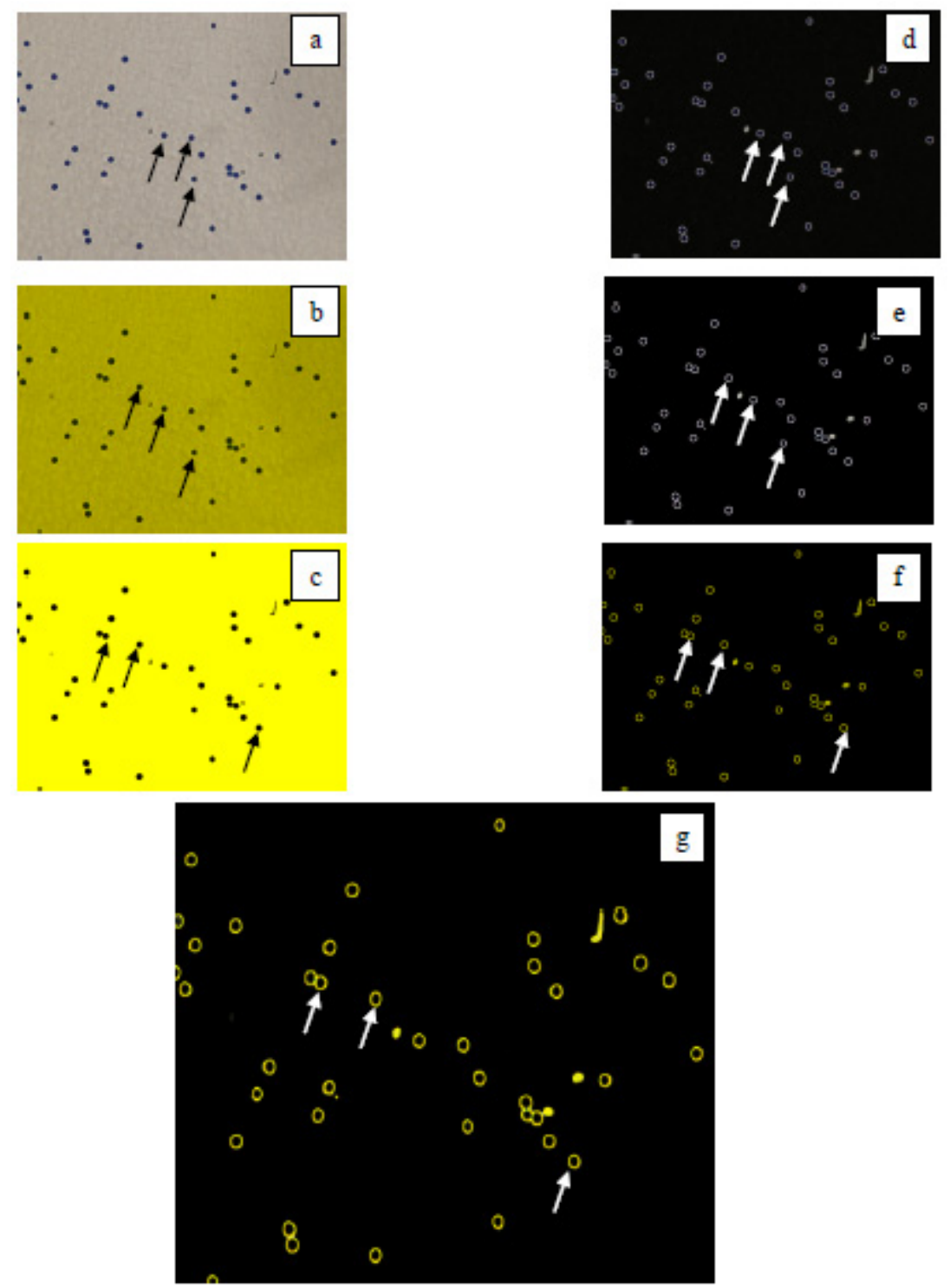

Figure 1. Stages during digital image processing prior to counting viable pollen with $\mathrm{a}-\mathrm{g}$ showing changes in one image through the processing steps. The blue circles (e.g. the black arrows in a-c) and open circles (e.g. the white arrows in $\mathrm{d}-\mathrm{g}$ ) depict viable pollen grains before and after digital image processing respectively

\subsection{Software Training for Viable Pollen Count}

For each new slide, the 'viable pollen count settings' was loaded from the previous step and the program was allowed to count all the grains that it could detect. Any pollen grain that was not detected automatically was then manually added until all stained pollen grains were detected on the new slide. Thus the measurement settings are refined for each processed slide throughout the training process and the new settings saved so that the 'viable pollen count settings' file is updated. The counts and the morphological measurements determined by the software for each image were then exported into a spreadsheet. A manual count was also performed for each image to obtain a comparable visual count.

\subsection{Image Processing for Inviable (Unstained) Pollen Counts}

Detecting inviable pollen grains is a bit of a challenge as they do not take up the stain making them appear transparent. Additional steps of digital processing are required to enumerate these unstained pollen grains. Using the original unprocessed image captured (Figure 1a and Figure 2a), the 'edge detect' function of the software was 
enabled to highlight the edges of both stained and unstained pollen grains. All the grains now appeared as open white shapes (Figure 1d and Figure 2b) in a black background. The contrast was then adjusted so that these shapes appeared thicker than before (Figure 1e and Figure 2c) and the background was altered to make the shapes yellow even as the background remained black (Figure $1 \mathrm{f}$ and Figure $2 \mathrm{~d}$ ). The above steps altered both the viable and inviable pollen grains in the image. The 'image morphology' function was then adjusted until the inviable pollen grains turned into solid yellow objects (Figure $1 \mathrm{~g}$ and Figure 2e) while the viable ones remained as open yellow objects allowing the software to clearly distinguish between filled (inviable) and open (viable) objects in the image. These settings were saved as 'inviable pollen count settings' and the software training was performed as described above. The morphological measurements of unstained pollen and the training counts were exported to a separate spreadsheet and a manual count of inviable pollen provided a comparable visual count of each image. The training steps were repeated with several distinct images until the software detected all stained and unstained pollen without the need for manual interference. Successful training for both species was achieved at the end of counting 1708 images from 52 pollen samples for B. napus and 3828 images from 72 pollen samples for C. heterophylla.
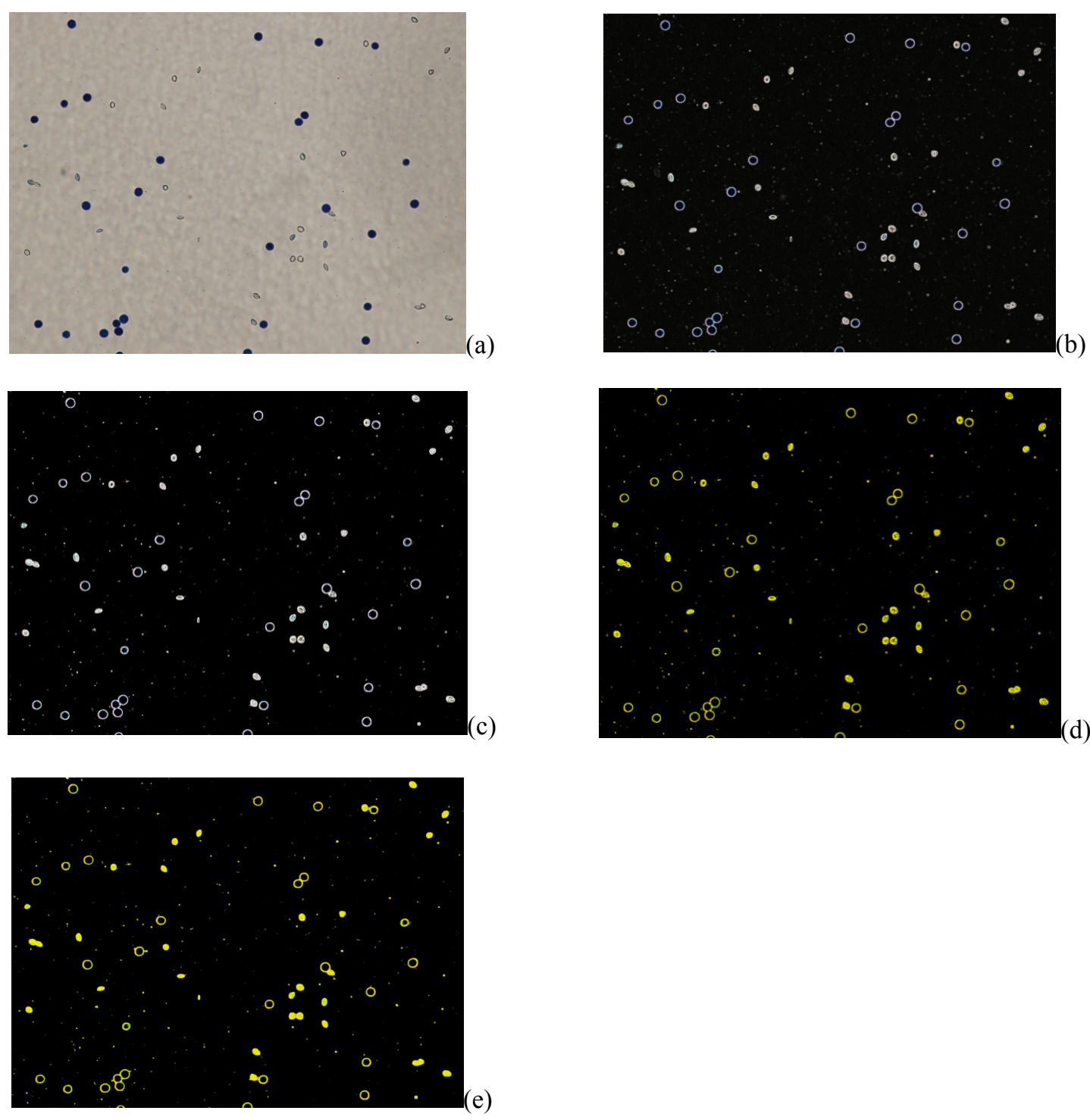

Figure 2. Inviable pollen grains of different shapes were picked up efficiently as seen in a - e showing changes to one image through the different processing steps. The white arrows in (e) show different shapes of inviable pollen grains_after digital processing 


\subsection{Software Testing}

For testing the efficiency of software performance, the respective 'object count settings' for stained and unstained pollen saved at the end of the training period was loaded and the software was allowed to obtain "test counts" for the same images used during training. No changes were made to the settings during the testing phase. The test counts were also exported into a different spreadsheet. In addition, new images were generated from freshly prepared slides. Pollen counts and viability estimates were first obtained using the software. Following this manual counts were performed on the same images.

\subsection{Statistical Analyses}

We performed a Pearson product moment correlation to detect the association between manual and software test counts of viable and inviable pollen grains and between software training and software test counts. Student's t-test compared the morphological measurements of viable and inviable pollen grains.

\section{Results and Discussion}

The total pollen per flower in Collinsia heterophylla was found to be an average of 56,461 $( \pm 52,489$ Standard Deviation (SD); $\mathrm{n}=72$ flowers), the proportion of viable pollen being $0.85 \pm 0.13(\mathrm{n}=72$ flowers). Flowers of Brassica napus had an average of 34,905 pollen grains ( \pm 43,930 SD; $\mathrm{n}=52$ flowers) and the proportion of viable pollen was $0.96( \pm 0.04 \mathrm{SD} ; \mathrm{n}=52$ flowers). The diameter of pollen grains ranged from $20-25 \mu \mathrm{M}$ in $C$. heterophylla and 31-37 $\mu \mathrm{M}$ in B. napus well within the range of 20-40 $\mu \mathrm{M}$ diameter predicted for a majority of angiosperm pollen (Muller, 1979). Pollen grains in both the species differed in their size and shape (Figure 3) with viable pollen being significantly larger in area as compared with the inviable pollen grains $(C$. heterophylla: $\mathrm{t}_{3826}=58.27, \mathrm{p}<0.0001 ; B$. napus: $\left.\mathrm{t}_{1706}=19.98, \mathrm{p}<0.0001\right)$. In addition, viable pollen grains were significantly more circular $\left(C\right.$. heterophylla: $\mathrm{t}_{3826}=10.05, \mathrm{p}<0.0001 ; B$. napus: $\left.\mathrm{t}_{1706}=8.99, \mathrm{p}<0.0001\right)$ and less elongate $(C$. heterophylla: $\mathrm{t}_{3826}=90.07, \mathrm{p}<0.0001 ; B$. napus: $\left.\mathrm{t}_{1706}=13.41, \mathrm{p}<0.0001\right)$. Using particle counters to distinguish viable and inviable grains is based on the shapes that can be identified by a fixed aperture setting (Kelly et al., 2002). However, our protocol overcomes the limitation of a fixed aperture setting by taking advantage of the ability of digital image processing to identify and target objects of any shape. We were able to detect the shapes of pollen grains in the image and distinguish them as viable and inviable pollen grains based on stain uptake making it more accurate than coulter counter estimates. Although the software has been set to use 5 measures for our counts, it is possible to include numerous different shapes to eventually count all the inviable pollen grains in any image. The staining protocol further strengthens our image-analysis approach as it is a histological method to determine pollen viability and aniline blue with its ability to stain callose is highly recommended for pollen grains (Heslop-Harrison et al., 1984). However, all viable pollen grains whether competitive on the stigmatic surface or not may take up stain providing a slightly exaggerated estimate of viability.

Pearson product moment correlation analyses between manual and software counts and between training and test counts by the software indicated high levels of similarity, which suggests that software counts are highly dependable for an accurate enumeraton of viable and inviable pollen. In both species viable (Figure 4) and inviable pollen grain (Figure 5) test counts were significantly correlated to manual and training counts. The software was also reliable when used with new images (Figure 6) where the software counts were done prior to manual counting. For viable pollen grains in C. heterophylla and B. napus, the correlation estimates were highly significant suggesting that the image processing protocol for viable pollen counts are very efficient. The correlation estimates for inviable pollen grains on the other hand differed between the species. The correlation estimates between test and training or manual counts were highly significant in both $C$. heterophylla $(\mathrm{r}=0.8$ to $0.9)$ and B. napus ( $\mathrm{r}=0.7$ to 0.8$)$. 

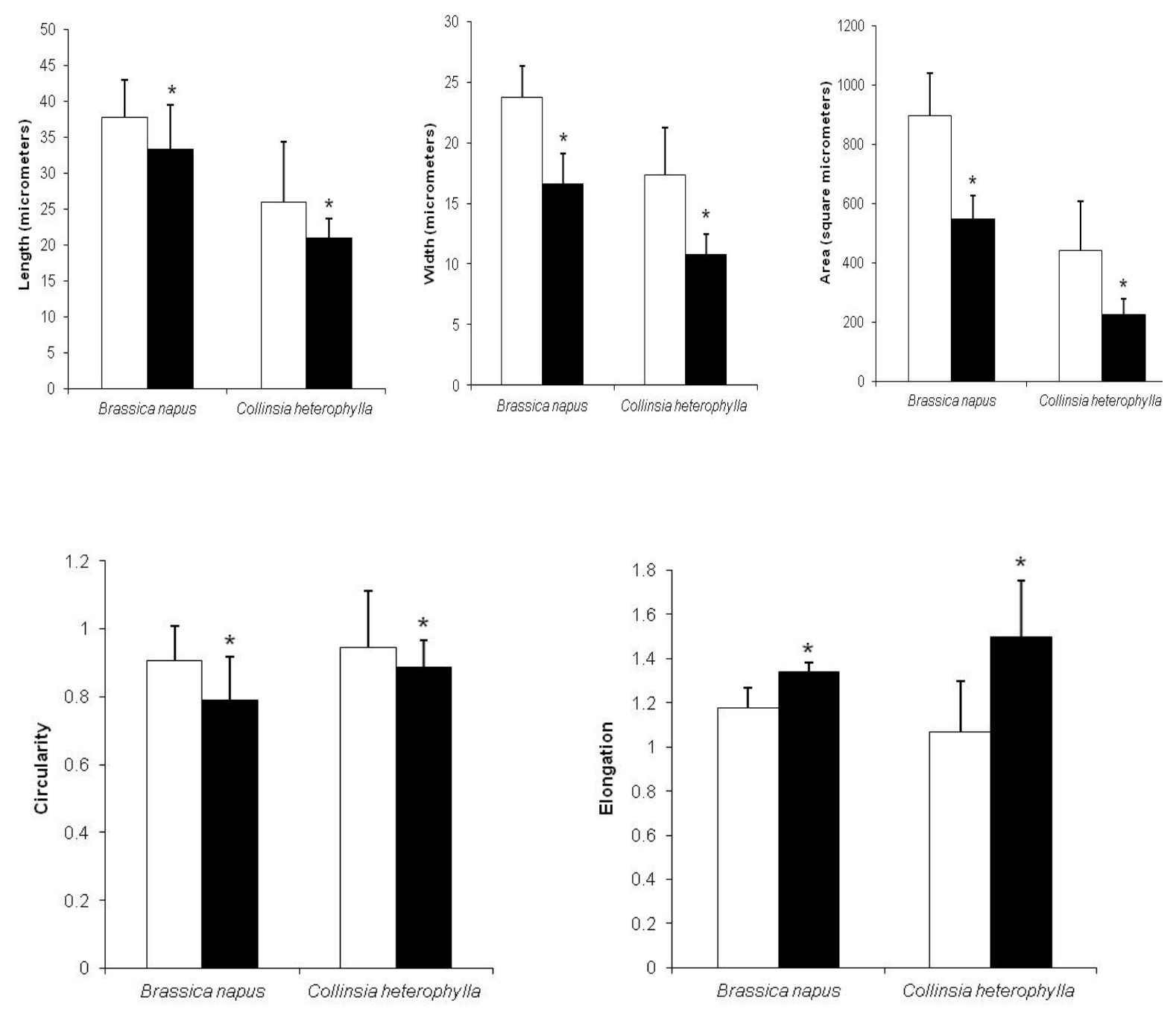

Figure 3. Comparison of measurements distinguishing viable and inviable pollen grains. Open bars represent viable pollen and closed bars depict measurements of inviable pollen grains. Statistical comparisons are between viable and inviable pollen grains within each species for the respective measures.

Significant differences are represented by * 

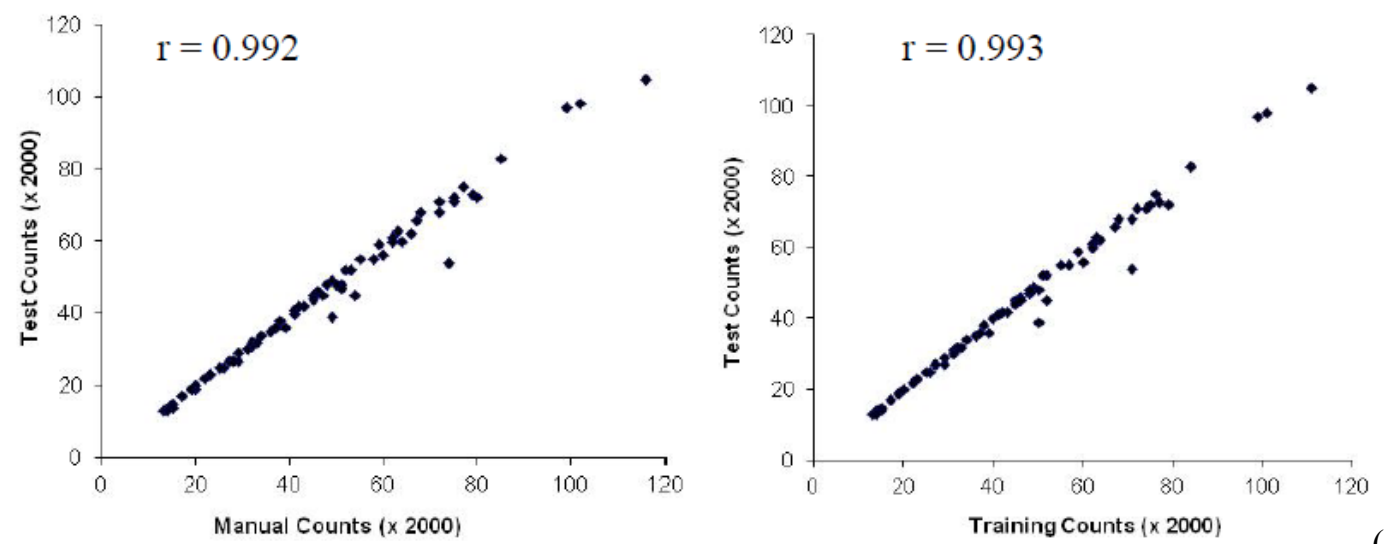

(a)
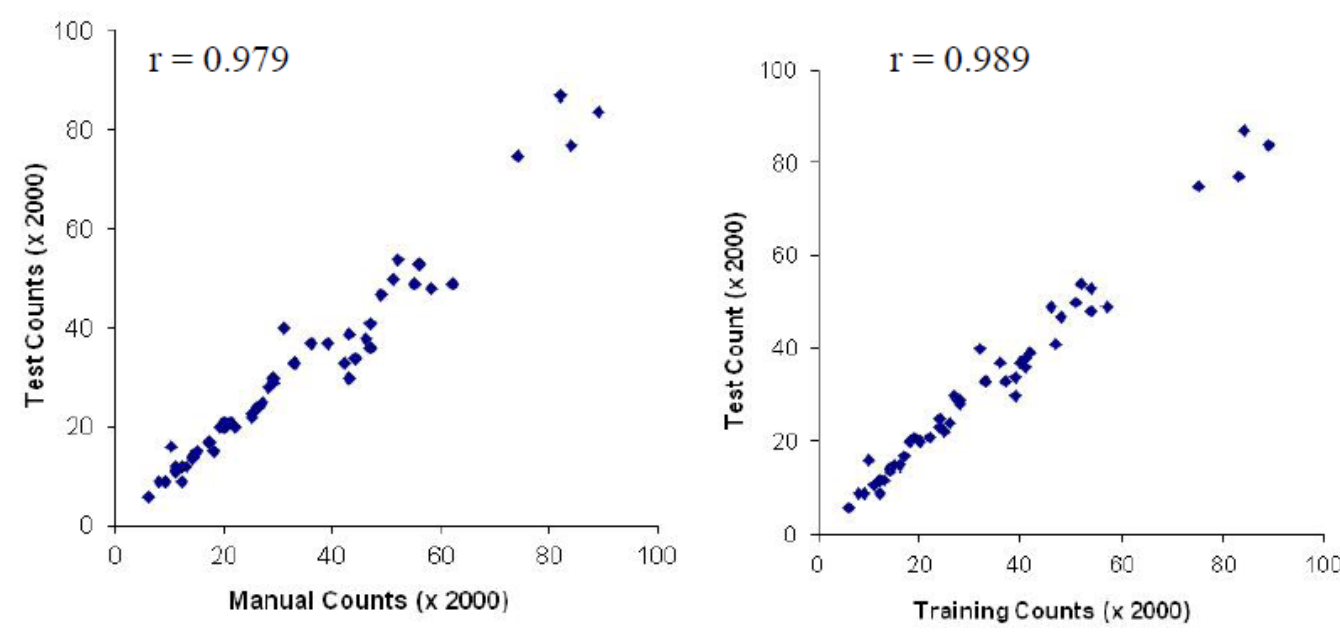

(b)

Figure 4. Relation between manual and software training counts with test counts of viable pollen grains of (a) Collinsia heterophylla (b) Brassica napus 

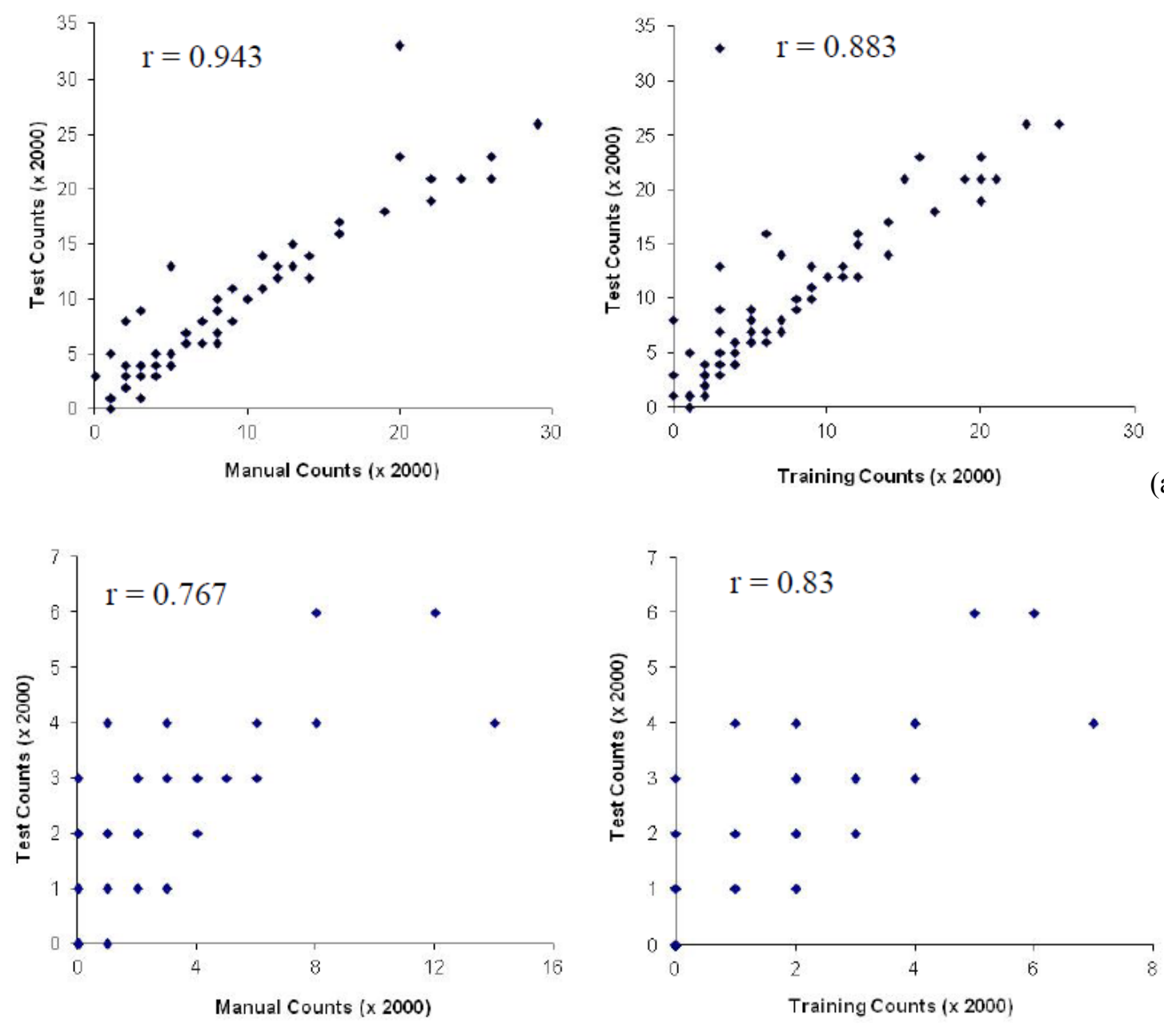

(a)

Figure 5. Relation between manual and software training counts with test counts of inviable pollen grains of (a) Collinsia heterophylla (b) Brassica napus
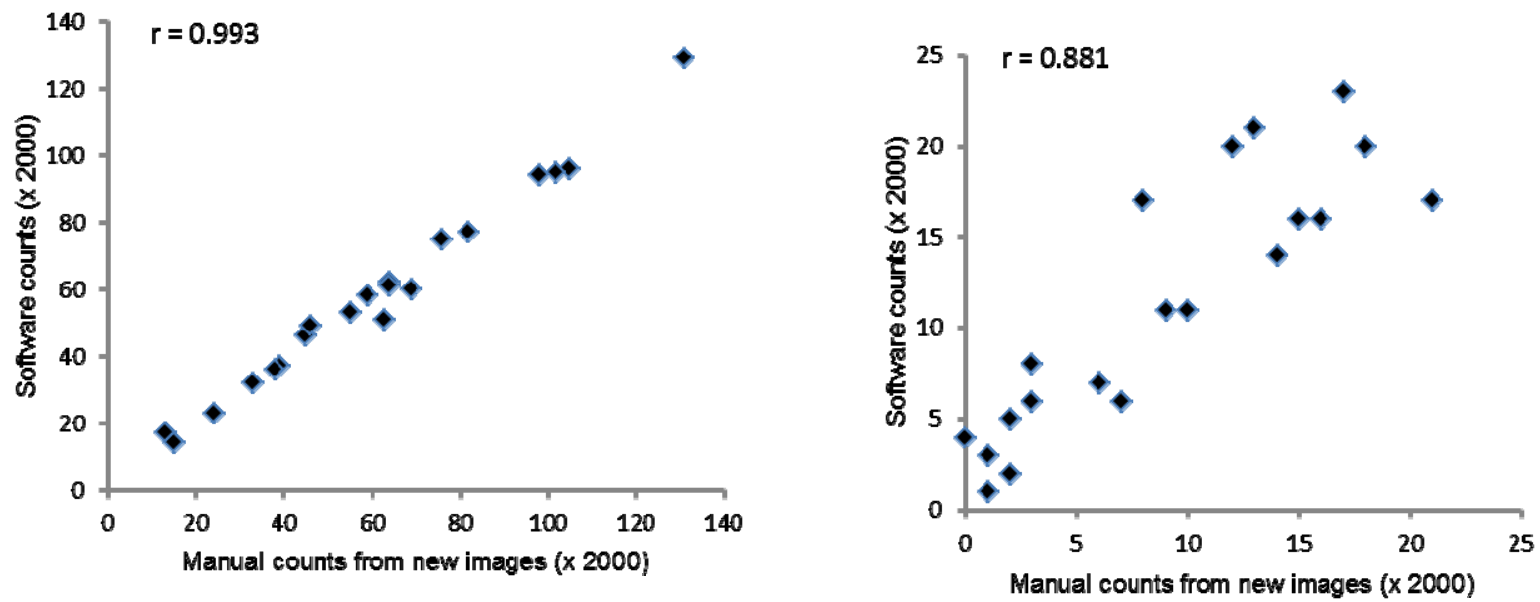

(a)

Figure 6. Relation between software and manual counts (a) viable and (b) inviable pollen grains with new images of Collinsia_heterophylla pollen grains 
One of the reasons for the lower correlation in B. napus could be due to increased tendency of pollen grains to cluster together making it difficult for the software to correctly count all the grains within each cluster. Clustering of pollen grains can be minimized by vortexing the centrifuge tubes immediately prior to drawing out the sample ensuring proper dispersal of pollen grains in the pollen-stain solution. If there are very few pollen grains, manual counting may be efficient especially when the grains tend to cluster as individual pollen grains can be delineated much more efficiently through a human eye. The imaging software is not capable of accurate delineation but the relatively high and significant correlation values support the fact that the software provides dependable estimates of inviable pollen grain numbers. In addition, plants of B. napus had very high pollen viability resulting in very few inviable pollen grains. There were many samples with few or no inviable pollen grains giving the discrete distribution of pollen grains seen in Figure 5b. Such low counts for inviable pollen grains could have been responsible for the observed spread of B. napus inviable pollen grains in our figures. Another trend that can be observed in Figure 5 is that the number of inviable pollen grains per flower in $C$. heterophylla was much higher than those in flowers of $B$. napus. This difference and higher pollen inviability between these two species could result from differences in growing conditions. While $B$. napus flowers were collected from plants grown under drought stress, $C$. heterophylla flowers were collected from plants grown under temperature stress i.e. higher than normal temperatures during the plant lifecycle. These plants were a part of another larger study to document the effect of climate change on plant reproduction. In plants, temperature stress has been shown to disrupt micro-gametogenesis resulting in higher incidences of pollen grain inviability (Hedhly, Hormaza, \& Herrero, 2009; Hedhly, 2011; Zinn, Tunc-Ozdemir, \& Harper, 2010) which explains why $C$. heterophylla has high numbers of inviable pollen grains per flower.

In conclusion, the accuracy of our protocol tends to increase when there are over a hundred pollen grains per image. It requires less effort when switching between different species, in contrast to the particle counter protocol, for which one has to generate preliminary size distributions for each species by staining and visual counting which are tedious in themselves. Kelly et al. (2002) recognizing this limitation in the Coulter counter method, mention that the effort to obtain size distributions is lesser than the effort required to count several samples on a haemocytometer. Our procedure, by eliminating the need for any counting with a haemocytometer is far superior to the Coulter counter method. Generating the viable and inviable pollen count settings for a new species by our method also takes much less effort. For a particle counter to provide an accurate estimate of pollen viability it is necessary to have little or no overlap in the size distributions of inviable and viable grains. To avoid erroneous counts using the particle counter, one must ensure that the size difference settings for viable and inviable grains is larger than the natural variation in the size of viable pollen in the species (Kelly et al., 2002). Our protocol is more accurate as it uses staining to distinguish between viable and inviable pollen grains. Inviable pollen grains come in diverse shapes and a particle counter cannot be set to detect many of these shapes. By using multiple measures to detect the shape of unstained objects in the image space, digital image processing counts almost all the inviable pollen grains in the image (Figure 2). The only other method that can count objects of different shapes is the tedious and time consuming process of manual visual counting. Although specific to Nikon microscopes, this digital image processing protocol has all the advantages associated with manual counting with lesser time and effort.

\section{Acknowledgements}

We are grateful for undergraduate research support scholarships to SM that made parts of this study possible. AHS would like to thank Colorado State University Research Support that helped funding the study and publication of the manuscript.

\section{References}

Aronne, G., Cavuoto, D., \& Eduardo, P. (2001). Classification and counting of fluorescent pollen using an image $\begin{array}{lllll}\text { analysis system. Biotechnology and Histochemistry, } & 76, & 35-40 .\end{array}$ http://dx.doi.org/10.1080/105202901100307117

Barrett, S. C. H. (2002). The evolution of plant sexual diversity. Nature Review and Genetics, 3, 274-284. http://dx.doi.org/10.1038/nrg776

Bechar, A., Gan-Mor, S., Vaknin, Y., Shmulevich, I., Ronen, B., \& Eisikowitch, D. (1997). An image-analysis technique for accurate counting of pollen on stigmas. New Phytologist, 137, 639-643. http://dx.doi.org/10.1046/j.1469-8137.1997.00867.x

Bino, R. J., Tuyl, J. M. V., \& De Vries, J. N. (1990). Flow Cytometric Determination of Relative Nuclear DNA Contents in Bicellulate and Tricellulate Pollen. Annals of Botany, 65, 3-8. 
Costa, C. M., \& Yang, S. (2009). Counting pollen grains using readily available, free image processing and analysis software. Annals of Botany, 104, 1005-1010. http://dx.doi.org/10.1093/aob/mcp186

Dafni, A., \& Firmage, D. (2000). Pollen viability and longevity: Practical, ecological and evolutionary implications. Plant Systematics and Evolution, 222, 113-132. http://dx.doi.org/10.1007/BF00984098

Dafni, A., Kevan, P. G., \& Husband, B. C. (2005). Practical Pollination Biology. Enviroquest Ltd., Ontario, Canada.

Fang, X., Turner, N. C., Yan, G., Li, F., \& Siddique, K. H. M. (2010). Flower numbers, pod production, pollen viability, and pistil function are reduced and flower and pod abortion increased in chickpea (Cicerarietinum L.) under terminal drought. Journal of Experimental Botany, 61, 335-345. http://dx.doi.org/10.1093/jxb/erp307

Fonseca, A. E., Westgate, M. E., \& Doyle, R. T. (2002). Application of fluorescence microscopy and image analysis for quantifying dynamics of maize pollen shed. Crop Science, 42, 2201-2206. http://dx.doi.org/10.2135/cropsci2002.2201

France, I., Duller, A. W. G., Duller, G. A. T., \& Lamb, H. F. (2000). A new approach to automated pollen analysis. Quaternary Science Reviews, 19, 537-546. http://dx.doi.org/10.1016/S0277-3791(99)00021-9

Hedhly, A., Hormaza, J. I., \& Herrero, M. (2009). Global warming and sexual plant reproduction. Trends in Plant Science, 14, 30-36. http://dx.doi.org/10.1016/j.tplants.2008.11.001

Hedhly, A. (2011). Sensitivity of flowering plant gametophytes to temperature fluctuations. Environmental and Experimental Botany, 74, 9-16. http://dx.doi.org/10.1016/j.envexpbot.2011.03.016

Herlihy, C. R., \& Delph, L. F. (2009). Selection Lines of SileneLatifolia (Caryophyllaceae) Differ in How Stress Affects Pollen Production. International Journal of Plant Sciences, 170, 1103-1108. http://dx.doi.org/10.1086/605872

Heslop-Harrison, J., Heslop-Harrison, Y., \& Shivanna, K. R. (1984). The evaluation of pollen quality, and a further appraisal of the fluorochromatic (FCR) test procedure. Theoretical and Applied Genetics, 67, 367-375. http://dx.doi.org/10.1007/BF00272876

Kearns, C. A., \& Inouye, D. W. (1993). Techniques for pollination biologists.University Press of Colorado, Boulder.

Kelly, J. K., Rasch, A., \& Kalisz, S. (2002). A method to estimate pollen viability from pollen size variation. American Journal of Botany, 89, 1021-1023. http://dx.doi.org/10.3732/ajb.89.6.1021

Muller, J. (1979). Form and Function in Angiosperm Pollen. Annals of Missouri Botanical Garden, 66, 593-632. http://dx.doi.org/10.2307/2398913

Prasad, P., Craufurd, P., \& Summerfield, R. (1999). Fruit number in relation to pollen production and viability in groundnut exposed to short episodes of heat stress. Annals of Botany, 84, 381-386. http://dx.doi.org/10.1006/anbo.1999.0926

Zinn, K. E., Tunc-Ozdemir, M., \& Harper, J. F. (2010). Temperature stress and plant sexual reproduction: uncovering the weakest links. Journal of Experimental Botany, 61, 1959-1968. http://dx.doi.org/10.1093/jxb/erq053 